\title{
Édito « Hydroécologie appliquée » Nam Theun 2
}

Le développement d'un projet hydroélectrique cristallise à lui seul de nombreux enjeux voire défis à concilier : techniques, humains, sociaux, sanitaires, environnementaux, territoriaux ou encore économiques. Rares sont les projets qui les ont intégrés d'une manière aussi forte et poussée que celui de Nam Theun 2 et qui peuvent se revendiquer d'être un modèle de développement durable. Nam Theun $2 \mathrm{a}$ été et fait toujours figure de précurseur à plusieurs titres.

Mais l'une des singularités de Nam Theun 2, voulue par EDF et ses partenaires, est d'avoir associé au projet un programme ambitieux de suivi environnemental, couplé à une implication forte de la communauté scientifique, au travers de projets de recherche appliquée voire fondamentale. Cette spécificité, peut-être insuffisamment connue, s'est inscrite dans une démarche de transparence et d'objectivité pour caractériser les évolutions et le comportement de milieux aux fonctionnements complexes, contribuant ainsi au savoir.

Pour ce faire, à l'initiative d'EDF et de NTPC, différents moyens humains et techniques ont été mis en place. Ainsi, le Département de la Qualité de l'Eau et de la Biodiversité de Nam Theun 2 a en charge la mise en œuvre et la réalisation du plan de gestion et de suivi environnemental du projet. II intègre un laboratoire qui assure les prélèvements et analyses en temps réels. Les compartiments étudiés sont la qualité des eaux, les gaz à effets de serre -GES-, l'hydrobiologie. D'autres programmes de recherches ont également été lancés en parallèle sur des problématiques spécifiques, par EDF en partenariat avec des grands instituts de recherche.

Les résultats des inventaires, suivis et travaux de recherche, conduits selon une approche pluridisciplinaire intégrée, ont permis la découverte de nouvelles espèces et la fourniture d'informations scientifiques et écologiques très précieuses. Ils ont également permis l'acquisition de connaissances essentielles favorisant une bien meilleure compréhension du fonctionnement et de l'évolution des écosystèmes aquatiques tant sur l'hydrodynamique du réservoir, la qualité de l'eau que sur la faune aquatique dans l'aménagement et en aval. Des travaux de recherche complémentaires ont porté sur les émissions de GES de la retenue et à l'aval de celleci, et plus précisément sur les différentes voies d'émissions dont certaines n'étaient que rarement étudiées. Un bilan des émissions nettes de GES a ainsi été réalisé sur cet aménagement situé en zone subtropicale. Enfin, un modèle numérique 3D de la qualité d'eau a été développé, rendant possible la prévision de l'évolution de la qualité d'eau, en tenant compte de différents scénarii d'usages du bassin versant, mais aussi l'estimation des émissions de gaz à effet de serre d'un réservoir de cette nature. 
Ces travaux exemplaires et colossaux voient leur mise en valeur méritée dans la publication de ce numéro spécial de la revue «Hydroécologie Appliquée ». Nul doute qu'ils susciteront un vif intérêt auprès de la communauté scientifique et des professionnels de l'hydroélectricité et qu'ils bénéficieront au rayonnement d'une hydroélectricité durable et responsable.

\section{Claude Nahon}

Directrice du Développement Durable et de l'Environnement du Groupe EDF

Souple, respectueuse de l'environnement, économiquement performante, I'hydraulique, première des énergies renouvelables fournit discrètement et avec efficacité, depuis des décennies près d'un cinquième de l'énergie mondiale et offre encore un formidable potentiel de développement des systèmes électriques, notamment dans les pays émergents. Pour autant, la réalisation d'un grand aménagement hydro-électrique n'est jamais neutre pour son environnement d'accueil et la connaissance et l'anticipation des impacts potentiels, en particulier sur les milieux aquatiques, sont indispensables à la réussite des projets. Dans ces domaines, bien des choses restent à comprendre et à apprendre du comportement de ces milieux complexes et chaque réalisation concrète doit être l'occasion d'enrichir notre connaissance et nos moyens de prévention ou d'action.

C'est ainsi que fort d'une première expérience vécue avec l'aménagement de Petit Saut en Guyane Française, EDF-DPIH a souhaité accompagner la réalisation et l'exploitation de la centrale hydroélectrique de Nam Theun 2 implantée au Laos, dans un contexte hydro-climatique similaire, d'un ambitieux programme de suivi et de recherche fondamentale, matérialisé par la mise en place d'une compétence interne au projet sur les sciences de l'environnement.

Créé à l'initiative d'EDF et NTPC ( Nam Theun Power Company), le Département de la Qualité de l'Eau et de la Biodiversité (WQB Dept.) est une plateforme originale qui propose une expertise environnementale pluridisciplinaire. II inclut un Laboratoire (AE Lab) permettant le suivi de domaines variés tels que l'hydrobiologie, la chimie de l'eau et les GES.

Les travaux de recherche et de suivis pluridisciplinaires réalisés dans le Laboratoire ont permis d'acquérir des connaissances fondamentales dans des domaines comme l'hydrodynamique du réservoir, l'évolution la qualité de l'eau et des communautés aquatiques du réservoir et des rivières en aval et surtout ont permis pour la première fois d'obtenir un bilan des émissions nettes de gaz à effet de serre en milieu subtropical. Les nombreux résultats ont également rendu possible la mise en place d'un modèle prédictif de la qualité de l'eau d'un réservoir artificiel. $\mathrm{Ce}$ modèle est un outil innovant, à même d'optimiser la gestion de la qualité de l'eau de futurs projets d'aménagements hydroélectriques. II fournit également une estimation fiable des émissions de GES par des réservoirs tropicaux. 
Les résultats produits, communiqués au travers de ce numéro spécial de la revue "Hydroécologie Appliquée ", fruit de 6 années de travail, sont à même de susciter un réel intérêt auprès des experts et managers de projets hydroélectriques actuels ou futurs.

Jean-François Astolfi

Directeur de la Division « Production et Ingénierie Hydraulique » d'EDF (DPIH, 2005-2014) 